\title{
DISPOSITIVO DE TRAÇÃO DO MEMBRO SUPERIOR PARA OSTEOSSÍNTESES INTRAMEDULARES BLOQUEADAS ANTERÓGRADAS DE FRATURAS DIAFISÁRIAS DE ÚMERO
}

\author{
UPPER LIMB TRACTION DEVICE FOR ANTEROGRADE INTRAMEDULLARY \\ LOCKED NAIL OF HUMERAL SHAFT FRACTURES
}

Mário Chaves Corrêa', Felipe Antônio Gomes², Daniel Campos Linhares², Lucas Braga Jacques Gonçalves³, José Carlos Souza Vilela ${ }^{3}$, Ronaldo Percopi de Andrade ${ }^{4}$

\section{RESUMO}

As fraturas diafisárias de fêmur e tíbia no adulto são, na sua maioria, tratadas cirurgicamente, geralmente através de osteossínteses intramedulares bloqueadas. Algumas fraturas diafisárias cominutivas e/ou muito desviadas podem representar um verdadeiro desafio técnico. As mesas de fraturas (ou ortopédicas), que permitem a estabilização instrumental vertical, horizontal e rotacional do membro, facilitam enormemente as manobras de redução e de colocação do implante e são amplamente utilizadas pelos cirurgiões ortopédicos. As fraturas diafisárias de úmero são, na sua maioria, tratadas não cirurgicamente. Entretanto, algumas requerem o tratamento cirúrgico, cujas indicações estão bem definidas na literatura. Podem ser fixadas através de placas ou de hastes intramedulares por via anterógrada ou retrógrada. No úmero, as manobras de redução da fratura e estabilização do membro para a implantação da haste intramedular são realizadas manualmente, geralmente por dois auxiliares e, por serem sujeitas à fadiga muscular, podem ser menos eficientes. $\mathrm{O}$ objetivo deste trabalho é apresentar um dispositivo externo de tração do membro superior para utilização em osteossínteses intramedulares bloqueadas anterógradas de fraturas diafisárias de úmero que permite a estabilização vertical, horizontal e rotacional do membro superior, de maneira similar àquela utilizada para os membros inferiores. O dispositivo é portátil, de construção simples, e pode ser instalado em qualquer mesa cirúrgica equipada com trilhos laterais. Foi utilizado no tratamento cirúrgico de 29 fraturas diafisárias de úmero com haste intramedular bloqueada anterógrada. Nossa experiência foi extremamente positiva. Não tivemos nenhuma complicação relacionada à sua utilização, que acreditamos ter facilitado, de maneira notável, os procedimentos cirúrgicos.

Descritores - Fraturas do úmero/cirurgia; Diáfise; Fixação intramedular de fraturas

\section{ABSTRACT}

Diaphyseal fractures of the femur and tibia in adults are mostly treated surgically, usually by means of intramedullary locked nail. Some comminuted and/or very deviated shaft fractures can represent a real technical challenge. The fracture table, which allows for the vertical, horizontal and rotational instrumental stabilization of the limb, greatly facilitates reduction and implant placement maneuvers and are widely used by orthopedic surgeons. Humeral shaft fractures are mostly treated nonsurgically. However, some whose indications are well defined in literature require surgical treatment. They can be fixed by plates, or by anterograde or retrograde intramedullary nail. In the humerus, limb fracture reduction and stabilization maneuvers for implantation of intramedullary nails are done manually, usually by 2 assistants. Because they are subject to muscle fatigue, this option may be less efficient. The aim of this paper is to present an external traction device for use in anterograde intramedullary fixation of humerus shaft fractures that allows vertical, horizontal and rotational stabilization of the upper limb similarly to that used in the lower limbs. The device is portable, of simple construction, and can be installed on any operating table equipped with side rails. It was used in the surgical treatment of 29 humeral shaft fractures with anterograde locked intramedullary nail. Our experience was extremely positive. We had no complications related to its use and we believe it to have facilitated the surgical procedures in a remarkable way.

Keywords - Humeral fractures/surgery; Diaphyses; Fracture fixation, intramedullary

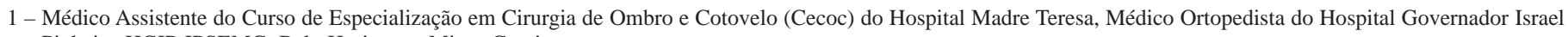
Pinheiro-HGIP-IPSEMG, Belo Horizonte, Minas Gerais.

2 - Médico Residente em Ortopedia e Traumatologia do HGIP-IPSEMG, Belo Horizonte, Minas Gerais.

3 - Médico Assistente do Curso de Especialização em Cirurgia de Ombro e Cotovelo (Cecoc) do Hospital Madre Teresa, Belo Horizonte, Minas Gerais.

4 - Chefe do Serviço de Ortopedia e Traumatologia do Hospital Madre Teresa, Belo Horizonte, Minas Gerais.

Trabalho realizado no Hospital Madre Teresa, Belo Horizonte, Minas Gerais.

Correspondência: Rua Chile, 245/404 - 30310670 - Belo Horizonte, MG - E-mail: mrccf@uol.com.br 


\section{MÉTODO}

O dispositivo foi utilizado no tratamento cirúrgico de 29 fraturas diafisárias de úmero, no período compreendido entre abril de 2005 e junho de 2009.

As indicações para o tratamento cirúrgico foram fraturas patológicas $(n=6)$, fraturas não preenchendo os critérios de desvio e encurtamento aceitáveis após tentativa de redução incruenta $(n=5)$, fraturas em pacientes obesos e/ou não cooperativos e/ou incapazes de tolerar o tratamento funcional com tutor do tipo Sarmiento (n $=8)$, fraturas em politraumatizados $(n=7)$ e retardes de consolidação $(n=3)$.

O dispositivo é constituído de três peças principais confeccionadas em aço inoxidável e pode ser esterilizado em autoclave: um suporte acolchoado para apoiar e fixar a cabeça do paciente, uma pequena mesa radiotransparente para apoiar o braço e o dispositivo de tração propriamente dito (Figuras 1a, 1b e 1c).

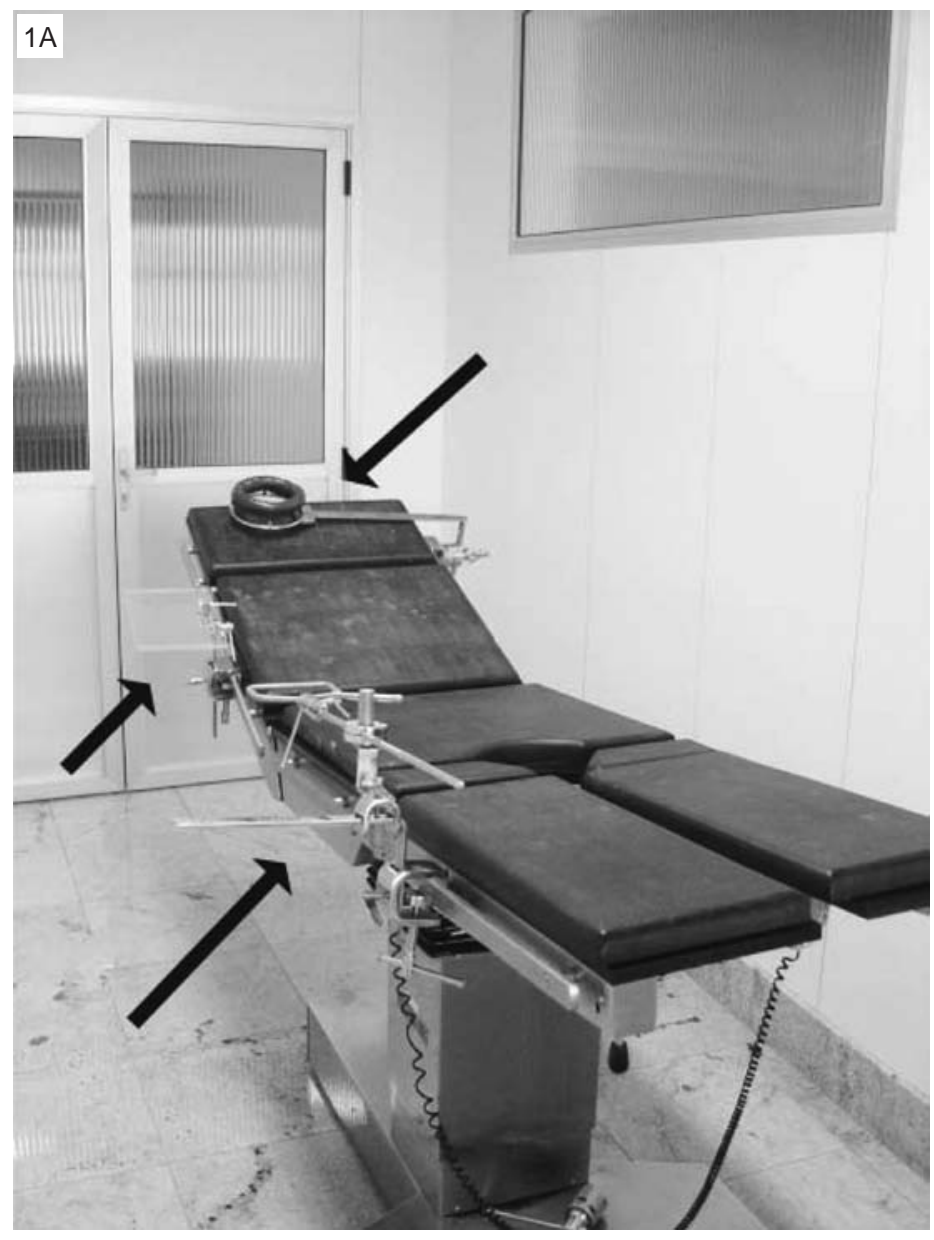

Figura 1A - Aspecto do dispositivo, constituído de três peças principais fixadas ao trilho lateral de uma mesa cirúrgica standard: um suporte para apoiar e fixar a cabeça, uma mesa radiotransparente e o dispositivo de tração propriamente dito. Figura 1B - Vista proximal do paciente posicionado para o procedimento. Figura 1C - Detalhe do dispositivo de tração adaptado ao trilho lateral distal da mesa cirúrgica

\section{TÉCNICA}

O paciente é colocado em decúbito dorsal na mesa cirúrgica para a administração da anestesia. O suporte acolchoado adaptado à extremidade proximal da mesa cirúrgica permite que o paciente seja colocado bem lateralmente à mesma para deixar livre toda a cintura escapular, facilitando o posicionamento do intensificador de imagens, que pode ser colocado paralelo, perpendicular ou oblíquo à mesa cirúrgica. A cabeceira é elevada entre $30^{\circ}$ e $40^{\circ}$ e a cabeça é fixada ao suporte, mantendo-a alinhada ao tronco para evitar o estiramento das estruturas neurovasculares. Opcionalmente, o paciente pode ser colocado em decúbito dorsal estrito. O braço do paciente é colocado sobre a pequena mesa radiotransparente, que dispõe de regulagem de altura e inclinação, adaptada ao trilho lateral intermediário da mesa cirúrgica (Figura 2). O dispositivo de tração é constituído por um suporte em forma de L invertido (“deitado”), que é fixado ao trilho
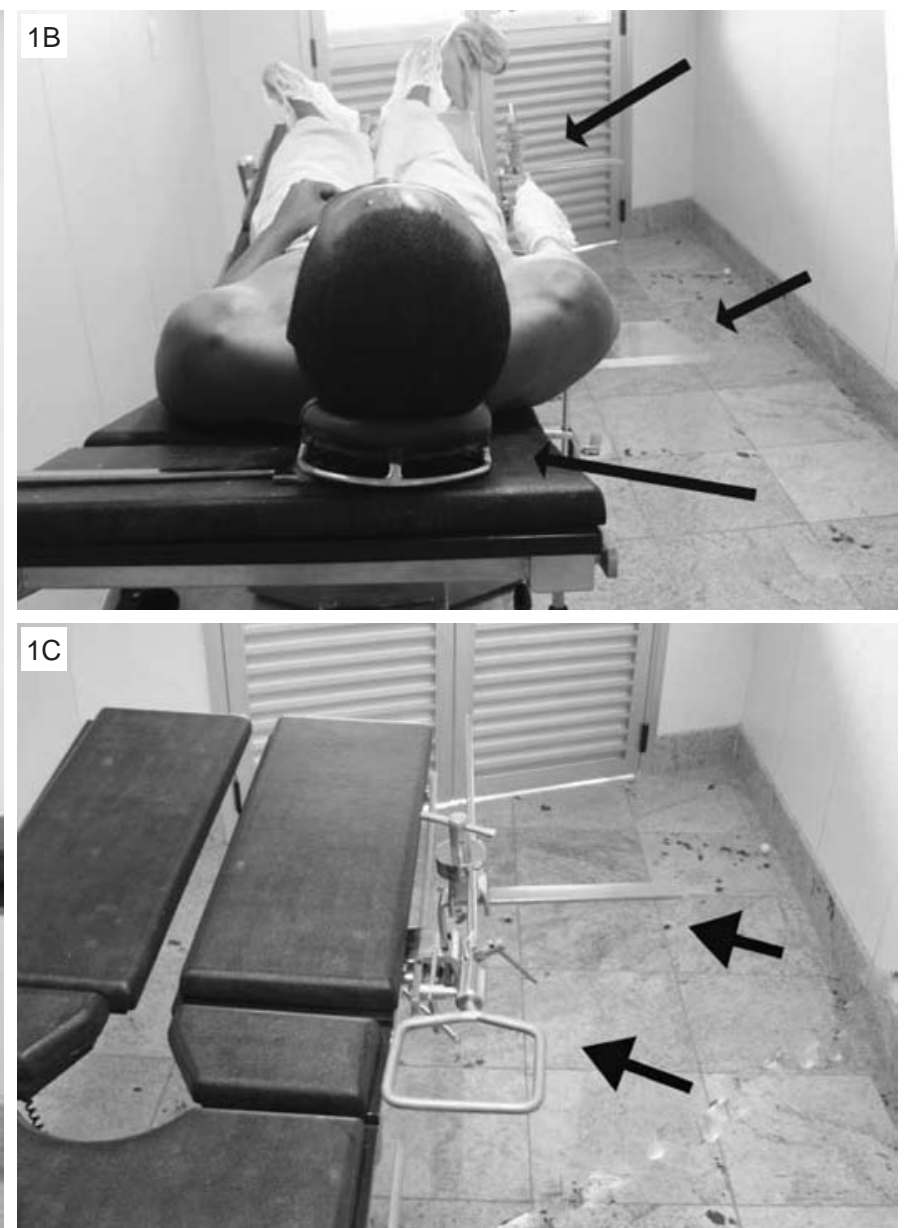


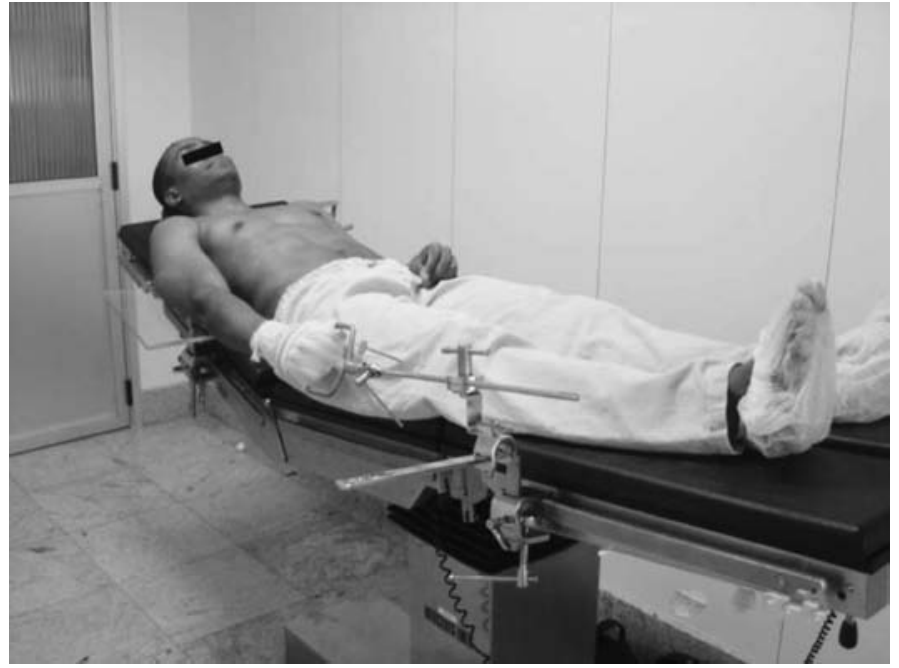

Figura 2 - Aspecto lateral do paciente posicionado para o procedimento

lateral distal da mesa e recebe uma peça que desliza sobre seu componente horizontal, podendo ser travada em qualquer ponto do mesmo. Essa peça, por sua vez, é dotada de um orifício pelo qual desliza longitudinalmente uma haste cilíndrica, que também pode ser travada em qualquer ponto. Esta haste conecta-se a um dispositivo em forma de estribo onde a mão do paciente é fixada por enfaixamento estéril. Assim, o membro superior, com o cotovelo estendido, pode ser tracionado longitudinalmente e/ou rodado em torno do próprio eixo e travado na posição desejada (Figura 3). Os movimentos de adução e abdução do membro superior ocorrem com o deslizamento da peça que prende a haste cilíndrica sobre o componente horizontal do suporte em L invertido. Os movimentos curtos de flexão e extensão do membro

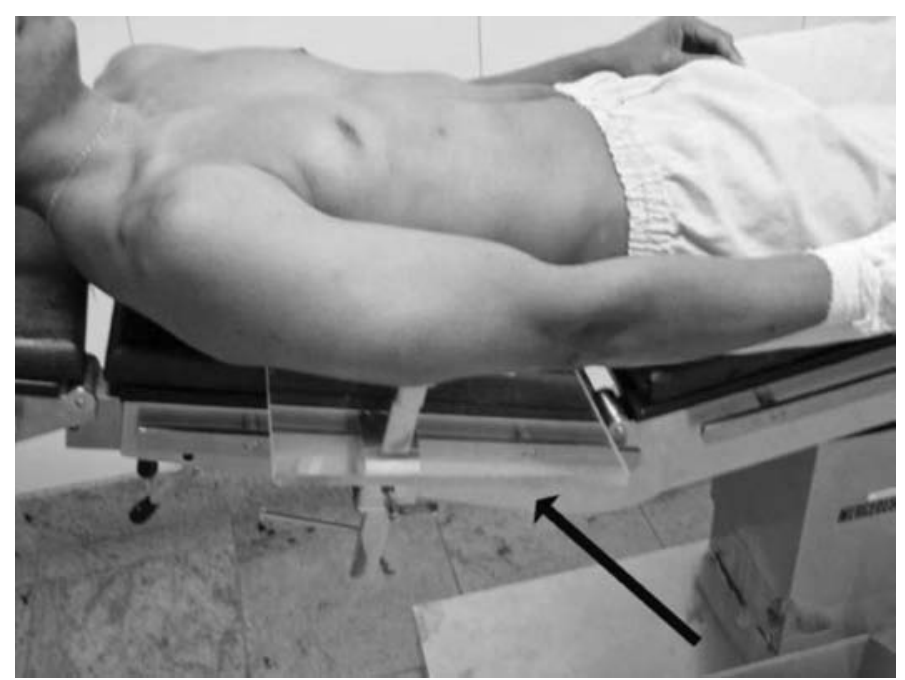

Figura 3 - Se for necessária a abertura do foco de fratura, a mesa pode ser usada como mesa cirúrgica superior são obtidos com o deslizamento em sentido superior e inferior (respectivamente teto e assoalho da sala cirúrgica) do suporte em L invertido em relação ao trilho lateral distal da mesa cirúrgica, onde também existe um mecanismo de travamento.

A abordagem do espaço subacromial para a introdução do fio-guia é realizada com o membro mantido em tração e adução, junto ao tórax. A quantidade de tração longitudinal é regulada manualmente pelo cirurgião, de maneira similar àquela realizada nos membros inferiores na mesa de fraturas. Esta manobra coloca o tubérculo maior em posição lateral e distal, e levemente anterior, ao acrômio anterolateral, facilitando a sua visualização direta e radioscópica (Figura 4). Com a ampla abertura do espaço subacromial obtida, o fio-guia, as fresas e o implante podem ser facilmente introduzidos de maneira atraumática e em linha com o canal medular do úmero (Figuras 5a, 5b e 5c).

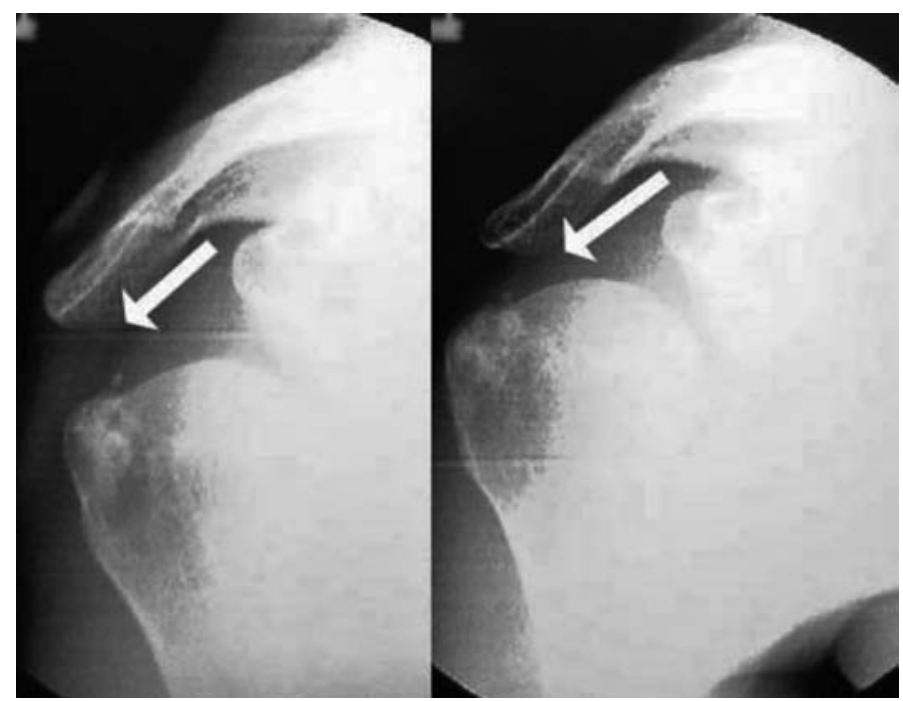

Figura 4 - Aspecto radioscópico do espaço subacromial. À esquerda, abertura obtida com o dispositivo de tração

Caso a tração instrumental e a manipulação dos fragmentos principais proximal e distal não sejam suficientes para a obtenção de alinhamento suficiente para permitir que o fio-guia alcance o canal medular do fragmento distal principal, o membro pode ser retirado do dispositivo e manipulado em todas as amplitudes de movimentos simples e combinados do ombro e do cotovelo e/ou ser hiperangulado ao nível do foco de fratura. A recolocação do mesmo novamente na tração pode ser feita, peroperatoriamente, sem dificuldade (Figura 6).

Desse modo o cirurgião dispõe de controle total do posicionamento do membro superior e pode ajustá-lo facilmente durante todo o procedimento. 

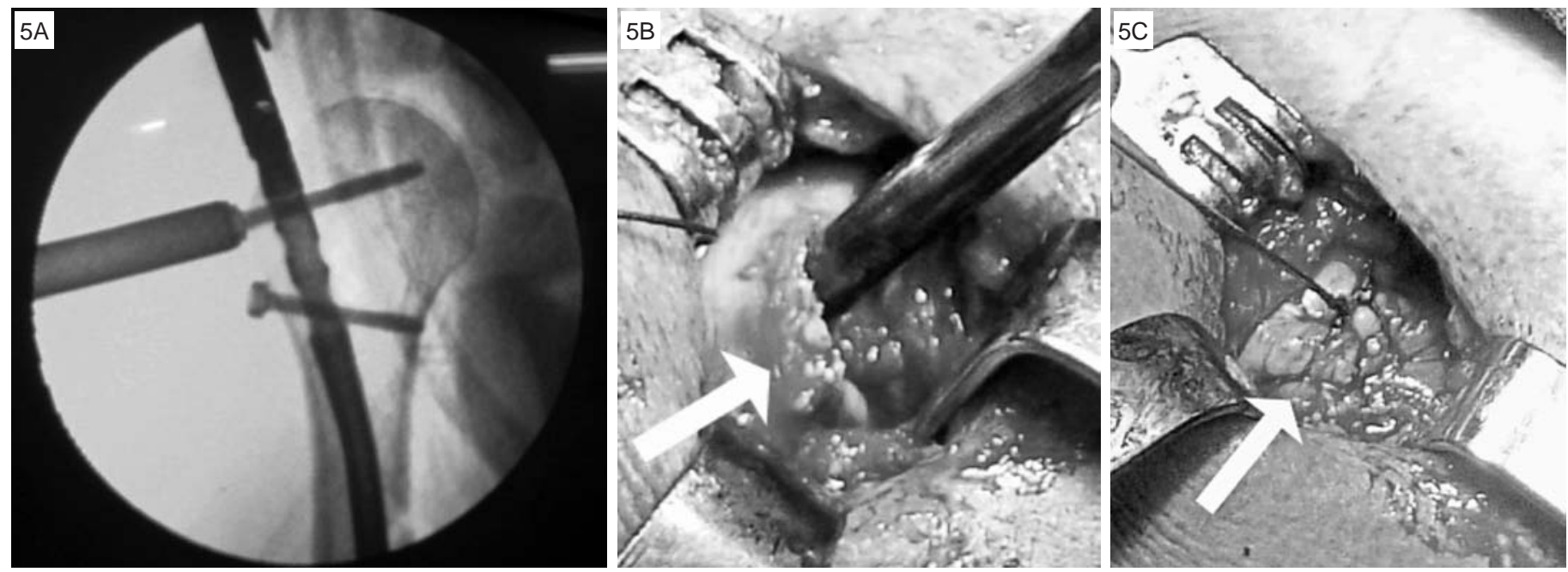

Figura 5A - Aspecto radioscópico da colocação dos parafusos de travamento proximal. Figura 5B - Aspecto da confecção do orifício de entrada na cabeça umeral, após a abertura do músculo supraespinhal. Figura $5 \mathbf{C}$ - Aspecto do supraespinhal reparado anatomicamente no fim do procedimento

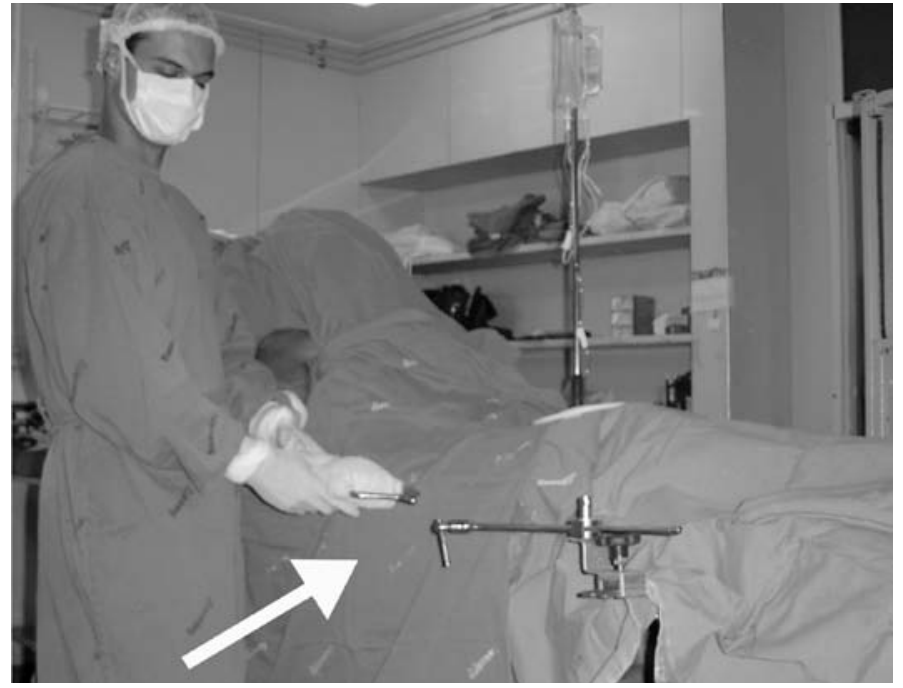

Figura 6 - Demonstração da facilidade de retirada peroperatória do dispositivo de tração, com técnica estéril
O braço tracionado fica apoiado sobre a mesa radiotransparente para estabilizá-lo nos sentidos horizontal (lateromedial) e vertical (superoinferior), respectivamente, facilitando a colocação dos parafusos de travamento proximais e distais, seja usando guias rígidos ou pela técnica free hand (Figuras 7a e 7b). Caso a abertura do foco de fratura seja necessária, a tração pode ser relaxada e a mesa utilizada como mesa cirúrgica (Figura 8).

\section{COMENTÁRIOS}

As fraturas diafisárias do úmero representam de $1 \%$ a $3 \%$ de todas as fraturas e, em sua grande maioria, podem ser tratadas com sucesso de maneira nãocirúrgica $^{(1,2)}$. O tratamento cirúrgico pode ser realizado
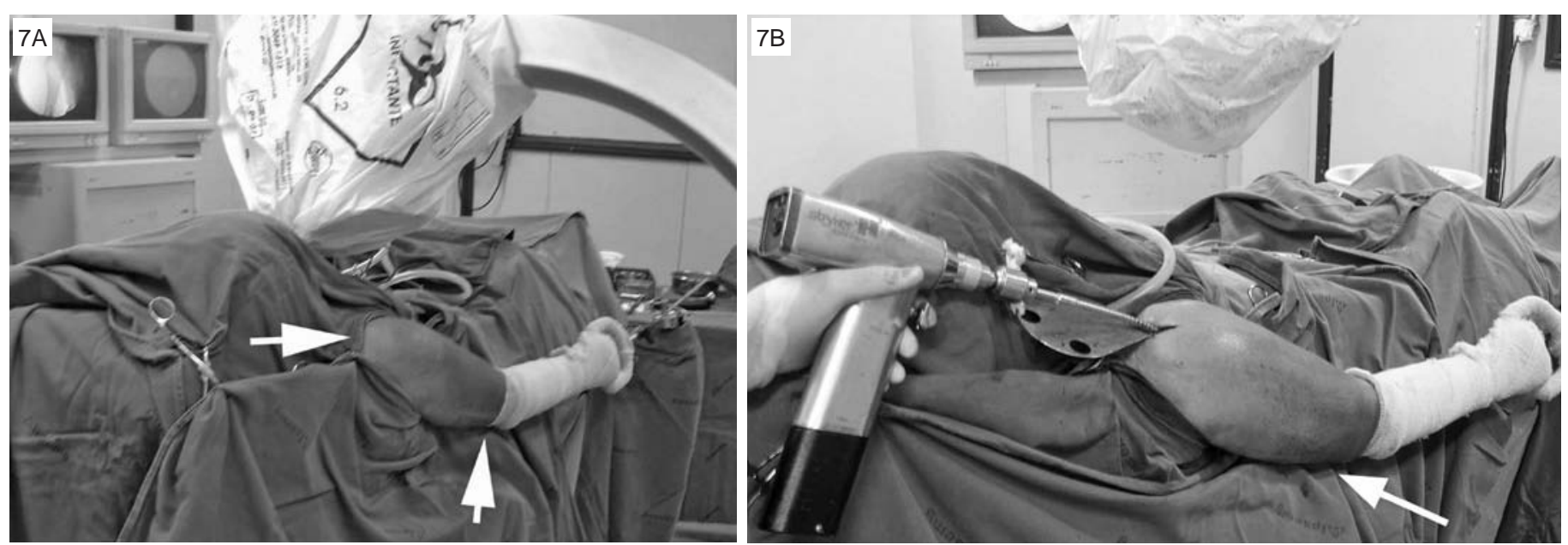

Figura 7A - Aspecto peroperatório. O intensificador de imagens pode ser colocado paralelo, perpendicular ou oblíquo à mesa de fraturas. Figura 7B - Aspecto peroperatório demonstrando o procedimento de fresagem do canal medular 


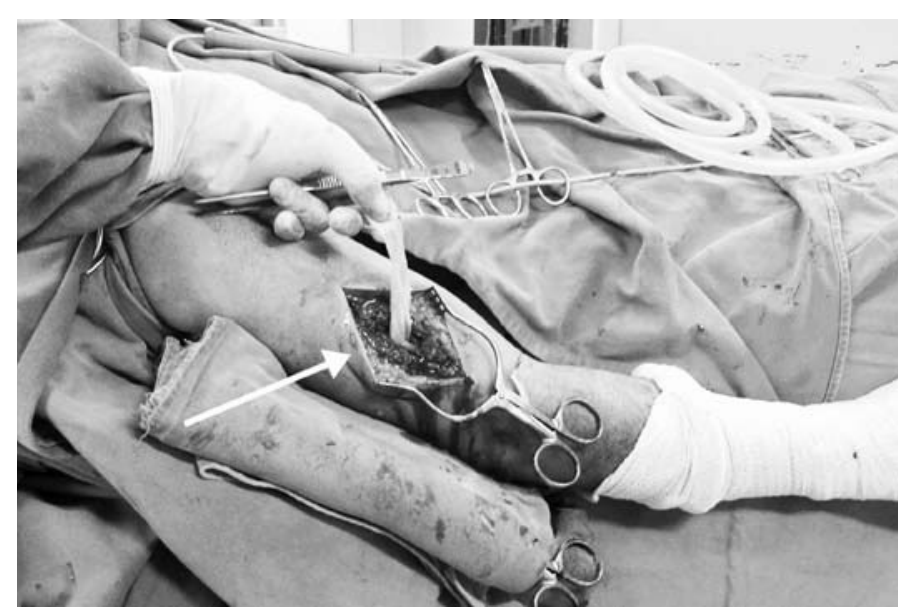

Figura 8 - Exploração do nervo radial. A tração é relaxada e a mesa radiotransparente é utilizada como mesa cirúrgica

através de redução aberta e fixação interna com placa ou de redução fechada e fixação intramedular bloqueada e está indicado em situações específicas: fraturas com desvios e/ou encurtamentos inaceitáveis após redução incruenta, em pacientes muito obesos, não cooperativos ou intolerantes ao tratamento funcional (Figuras 9a, 9b, 9c e 9d), na maioria das fraturas patológicas, em pacientes politraumatizados, nas fraturas expostas, segmentares ou bilaterais, no cotovelo flutuante ou naquelas associadas a lesão vascular requerendo reparo cirúrgico $^{(1-3,5)}$.
As fraturas diafisárias cominutivas e/ou muito desviadas dos ossos longos dos membros superiores e inferiores podem representar verdadeiros desafios técnicos para o cirurgião. A tração longitudinal do membro facilita o alinhamento e a redução dos fragmentos da fratura $^{(4)}$.

Em reduções abertas e fixações internas com placa e parafusos dos ossos longos dos membros superiores e inferiores, a tração, quando necessária, pode ser obtida através do uso de fixadores externos peroperatórios temporários ${ }^{(4)}$.

Em reduções fechadas e fixações internas com hastes intramedulares em fraturas diafisárias de fêmur e tíbia, a tração é tradicionalmente obtida com a mesa de fraturas, amplamente utilizada pelos cirurgiões ortopédicos. Nas osteossínteses intramedulares de fraturas diafisárias de úmero, a tração é normalmente realizada manualmente por um ou dois auxiliares ${ }^{(5)}$. Por ser sujeita à fadiga muscular, pode ser menos eficiente.

Na fixação intramedular por via anterógrada, a introdução do fio-guia, das fresas e, finalmente, da haste, deve ser feita de maneira precisa através da divulsão do músculo supraespinhal na área adjacente à sua inserção no tubérculo maior. É muito importante a visualização adequada do espaço subacromial. Desta maneira, o implante pode ser introduzido na posição correta, mini-
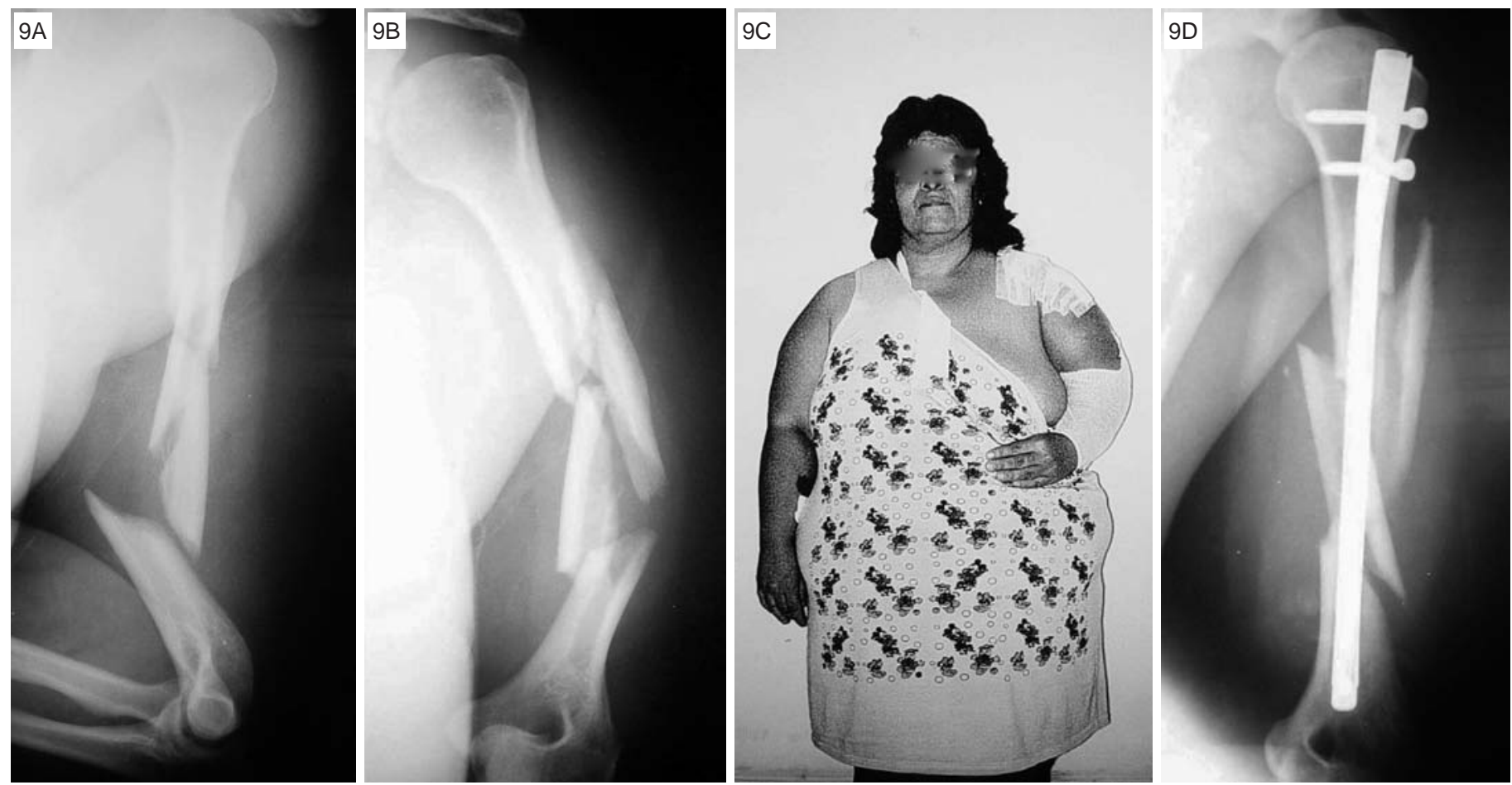

Figuras 9 A, B, C, D - Fratura cominutiva segmentar de úmero em paciente portadora de obesidade mórbida, fixada com haste de Seidel 
mizando o traumatismo ao músculo e permitindo o seu reparo no fim do procedimento, além de evitar a fratura do tubérculo maior, complicações que podem ser catastróficas para a função do ombro ${ }^{(1,3,5-13)}$.

A passagem do fio-guia para a fresagem do canal medular e introdução da haste é facilitada pelo alinhamento da fratura através da tração longitudinal do úmero. Algumas vezes são necessárias a manipulação e, eventualmente, a hiperangulação do foco de fratura para permitir que o fio-guia alcance o canal medular do fragmento distal principal. O desvio rotacional da fratura, se presente, deve ser corrigido após a passagem do fio-guia e antes da fresagem do canal medular.

O dispositivo ora descrito permite excelente exposição do espaço subacromial, o que auxilia na introdução precisa e atraumática da haste intramedular.

O membro pode ser mantido em tração e bloqueado em qualquer grau de rotação de maneira estável, e também ser retirado da tração e manipulado quantas vezes forem necessárias.

O posicionamento estável do membro superior nos sentidos vertical e horizontal facilita a colocação dos parafusos de travamento proximais e distais da haste intramedular, que podem ser feitos através de guias rígidos ou pela técnica free hand. A abertura do foco de fratura, se necessária (por exemplo, quando não é possível passar o fio-guia pela técnica fechada, como pode ser o caso em retardes de consolidação, ou para

\section{REFERÊNCIAS}

1. Wolf JM, Athwal GS, Shin AY, Dennison DG. Acute trauma to the upper extremity: what to do and when to do it. J Bone Joint Surg Am. 2009;91(5):1240-52.

2. Anglen JO, Archdeacon MT, Cannada LK, Herscovici D Jr. Avoiding complications in the treatment of humeral fractures. J Bone Joint Surg Am. 2008;90(7):1580-9.

3. Chapman JR, Henley MB, Agel J, Benca PJ. Randomized prospective study of humeral shaft fracture fixation: intramedullary nails versus plates. J Orthop Trauma. 2000;14(3):162-6.

4. Boykin RE, Baskies MA, Harrod CC, Jupiter JB. Intraoperative distraction in the upper extremity. Tech Hand Up Extrem Surg. 2009;13(2):75-81.

5. Lin J, Hou SM. Locked nailing of severely comminuted or segmental humeral fractures. Clin Orthop Relat Res. 2003;(406):195-204.

6. Gaultier O, Rebai L, Dunaud JL, Moughabghab M, Benaissa M. Treatment of humeral shaft fractures with the seidel nail. A study of 23 cases with rotator cuff evaluation J Bone Joint Surg Br. 2000;82(Suppl 2):118.

7. Johnson EC, Strauss E. Recent advances in the treatment of gunshot fractures of the humeral shaft. Clin Orthop Relat Res. 2003;(408):126-32.

8. Blum J, Janzing H, Gahr R, Langendorff HS, Rommens PM. Clinical perfor- exploração do nervo radial), pode ser feita com o relaxamento da tração, com o braço apoiado sobre a mesa radiotransparente.

A compressão axial manual do foco de fraturas transversas ou oblíquas curtas pode ser obtida pela retirada do membro do dispositivo após a colocação dos parafusos de travamento proximais e antes da colocação dos distais.

O dispositivo funciona como uma mesa de fraturas semelhante às utilizadas nos membros inferiores. Permite a tração intermitente ou contínua do membro e auxilia na redução e fixação de fraturas diafisárias de úmero com haste intramedular anterógrada. O intensificador de imagens pode ser facilmente posicionado paralelo, perpendicular ou oblíquo à mesa de fraturas. Quando não é necessária a tração, o dispositivo funciona como um arm holder, facilitando o trabalho do cirurgião.

A aplicação do dispositivo de tração nas fixações intramedulares bloqueadas anterógradas de fraturas diafisárias de úmero, derivada da nossa experiência com o uso do mesmo em cirurgias artroscópicas e abertas de ombro na posição semiassentada (cadeira de praia) ${ }^{(14)}$, foi extremamente útil e facilitou enormemente a redução e a fixação das fraturas. Não tivemos nenhuma complicação relacionada à sua utilização e o empregamos rotineiramente nas osteossínteses intramedulares bloqueadas anterógradas de fraturas diafisárias de úmero. mance of a new medullary humeral nail: antegrade versus retrograde insertion. J Orthop Trauma. 2001;15(5):342-9.

9. Schoots IG, Simons MP, Nork SE, Chapman JR, Henley MB. Antegrade locked nailing of open humeral shaft fractures. Orthopedics. 2007;30(1):49-54.

10. Cox MA, Dolan M, Synnott K, McElwain JP. Closed interlocking nailing of humeral shaft fractures with the Russell-Taylor nail. J Orthop Trauma. 2000;14(5):349-53.

11. Scheerlinck T, Handelberg F. Functional outcome after intramedullary nailing of humeral shaft fractures: comparison between retrograde Marchetti-Vicenzi and unreamed AO antegrade nailing. J Trauma. 2002;52(1):60-71.

12. Cheng HR, Lin J. Prospective randomized comparative study of antegrade and retrograde locked nailing for middle humeral shaft fracture. J Trauma. 2008;65(1):94-102.

13. Lin J, Shen PW, Hou SM. Complications of locked nailing in humeral shaft fractures. J Trauma. 2003;54(5):943-9.

14. Correa MC, Gonçalves LB, Andrade RP, Carvalho LH Jr. Beach chair position with instrumental distraction for arthroscopic and open shoulder surgeries. J Shoulder Elbow Surg. 2008;17(2):226-30. 